\title{
Nutritionists educate Vietnamese immigrants about breastfeeding
}

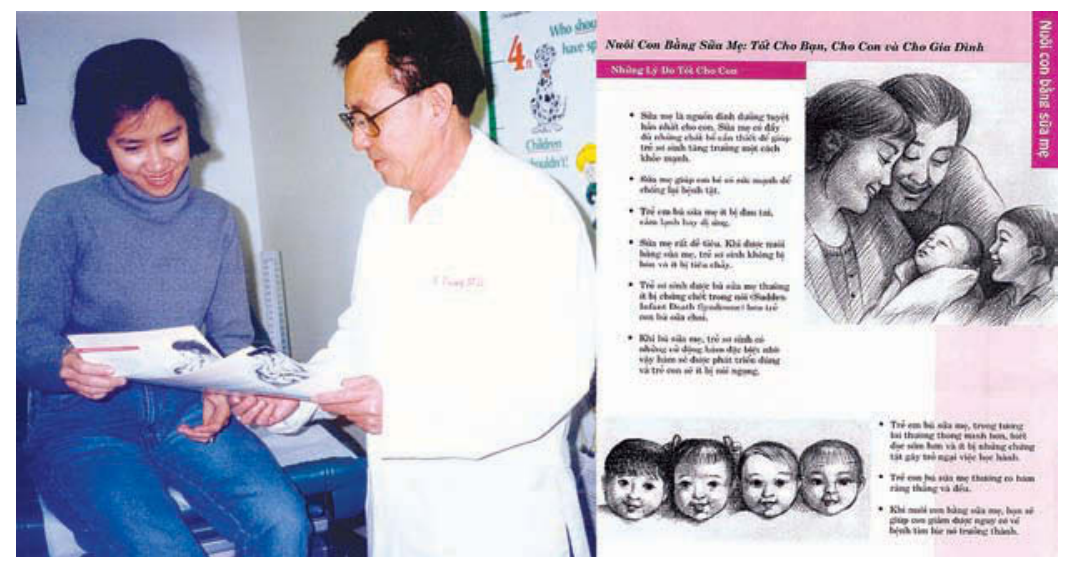

lthough breastfeeding is healthier for babies A and mothers, Southeast Asian women who immigrate to the West are far more likely to bottle-feed due to language and cultural barriers.

"They almost never see anyone breastfeeding," says Joanne Ikeda, UC Cooperative Extension (UCCE) nutrition specialist, who developed an intervention to promote breastfeeding among Vietnamese immigrants. "Most U.S. women seek privacy for breastfeeding, unlike other cultures where it is done more openly in public."

To help overcome barriers to breastfeeding, Ikeda and Kim-Phuc Nguyen, UCCE research nutritionist, adopted a three-pronged approach: they developed educational materials tailored to Vietnamese women; provided in-service training to physicians on the importance of breastfeeding; and distributed these materials for doctors to use with their pregnant patients. Giao Pham, a physician provider liaison with Blue Cross of California, was a consultant to the project.

Since doctors are held in high esteem in Vietnamese-American communities, they have a lot of influence with their patients. "They can help increase the low rates of breastfeeding by advising mothers to do so," Nguyen says.

The American Academy of Pediatrics recommends breastfeeding babies for a year, in part because breast milk contains antibodies that help protect infants from ear and respiratory diseases, as well as gastrointestinal infections. Breastfeeding also benefits mothers by, for example, reducing the risks of osteoporosis, and breast and ovarian cancers.

While Southeast Asians typically breastfeed in their native countries, this ethnic group has the lowest breastfeeding rate in California. Between 1997 and 1999 only $20 \%$ of the state's Vietnamese mothers breastfed their newborns at the hospital, compared with $62 \%$ of white mothers, according to a California Department of Health Services report. "There is no
Far left, Hien Truong, a San Jose obstetrician, shares breastfeeding information with a pregnant patient. Left, UC Cooperative Extension developed six brochures targeted to reach Vietnamese immigrants, whose breastfeeding rates are among the lowest of California ethnic groups.

choice in Vietnam but here they say 'Why breastfeed? Everything is all ready'," Nguyen says.

Many Southeast Asian immigrants also believe that formula is superior to breast milk, because their breastfed Asia-born infants were more likely to die than their U.S.-born, bottle-fed infants. Other factors in the decision to bottle-feed include the fear of not making enough milk and the pressures of going back to work, Nguyen says.

\section{Prenatal education evaluated}

Prenatal education and support are the best ways to increase breastfeeding rates, Ikeda says. To help educate Vietnamese immigrants, UCCE collaborated with the Vietnam Physician's Association of Northern California to develop six breastfeeding pamphlets in their native language. The pamphlets explain the health benefits, dispel myths and offer advice on overcoming problems.

Equally important, "the educational materials are culturally appropriate," Nguyen says. For example, because many Southeast Asians are lactoseintolerant, the materials advise nursing mothers to eat calcium-rich foods such as sardines and tofu rather than milk and cheese.

As a component of a pilot program to evaluate the effectiveness of this approach, in 1999 the educational materials were distributed to 11 Vietnamese physicians, who in turn gave them to Vietnamese women during prenatal examinations. In an unpublished survey of 78 mothers who received the educational materials, the researchers found that the breastfeeding rate was initially high but then dropped off sharply. While more than $90 \%$ of the mothers breastfed in the hospital, the rate fell to $60 \%$ at 4 weeks, about $20 \%$ at 4 months and $15 \%$ at 6 months. The results suggest that mothers need more education and support after leaving the hospital, Nguyen says.

The materials are available online and are being used by health organizations around the country, including Kaiser in Oakland and the U.S. Department of Agriculture's Women, Infants and Children (WIC) program.

The pilot program "sought to demonstrate that convincing physicians of the importance of breastfeeding, and having them promote this practice with their patients, can increase breastfeeding rates among Southeast Asian women in California," Ikeda says. - Robin Meadows 